\title{
Perspective
}

PERSPECTIVE Actualité en histoire de l'art

1 | 2012

Art et pouvoir

\section{Compromission, engagement, neutralité : analyses de l'art polonais de la guerre froide}

Submission, engagement, neutrality: analyses of Polish art from the Cold War

\section{Mathilde Arnoux}

\section{(2) OpenEdition}

1 Journals

Édition électronique

URL : http://journals.openedition.org/perspective/657

DOI : $10.4000 /$ perspective. 657

ISSN : 2269-7721

Éditeur

Institut national d'histoire de l'art

\section{Édition imprimée}

Date de publication : 30 juin 2012

Pagination : 193-200

ISSN : 1777-7852

\section{Référence électronique}

Mathilde Arnoux, «Compromission, engagement, neutralité : analyses de l'art polonais de la guerre froide », Perspective [En ligne], 1 | 2012, mis en ligne le 30 décembre 2013, consulté le 01 octobre 2020. URL : http://journals.openedition.org/perspective/657 ; DOI : https://doi.org/10.4000/ perspective.657 


\section{Compromission, engagement, neutralité : analyses de l'art polonais de la guerre froide}

Mathilde Arnoux

Depuis une vingtaine d'années, des expositions comme Europa, Europa: Das Jahrhundert der Avantgarde in Mittel- und Osteuropa, organisée à Bonn en 1994, Der Riss im Raum: Positionen der Kunst seit 1945 in Deutschland, Polen, der Slowakei und Tschechien, présentée à Berlin en 1995, et Les Promesses du passé : une histoire discontinue de l'art dans l'ex-Europe de l'Est, montrée à Paris en $2010^{1}$, ont mis en valeur la variété des pratiques artistiques dans les pays socialistes et l'importance d'envisager les différentes scènes européennes de manière distincte. Si l'on se penche sur le cas particulier de l'art polonais durant la guerre froide, il faut souligner que dans les synthèses récentes sur l'histoire de l'art de la seconde moitié du XX $\mathrm{X}^{\mathrm{e}}$ siècle, comme celles réalisée par Anda Rottenberg, et celle menée sous la direction de David Crowley ${ }^{2}$, la guerre froide est réintégrée dans une histoire plus longue. Ces ouvrages présentent les personnalités et les tendances artistiques polonaises de la seconde moitié du $\mathrm{XX}^{\mathrm{e}}$ siècle dans un contexte politique et social brossé à grands traits. L'organisation institutionnelle et ses évolutions ainsi que les directives officielles et leurs conséquences sur l'art, sont le cadre dans lequel les auteurs décrivent les événements marquants de la vie artistique, analysent les œuvres et identifient les thèmes récurrents dans les arts plastiques polonais des avant-gardes à aujourd'hui.

Si la politique artistique du pouvoir socialiste a été l'enjeu d'études permettant d'en comprendre le fonctionnement, le rapport entre l'art et le pouvoir ne constitue pas un sujet plus développé que pour les autres périodes. Dans ces travaux, le pouvoir politique de la période socialiste est présenté comme ayant déterminé le champ artistique en matière de sujets, d'expositions et d'échanges avec l'étranger. L'absence d'un marché de l'art libre a eu des conséquences sur la production des œuvres et sur les objectifs auxquels elle répondait, sur la façon dont les artistes et les critiques dialoguaient. À la mise au pas de l'art par le réalisme socialiste de 1949 à 1954 a succédé la période du dégel qui, dès le milieu des années 1950, a mis à mal la doctrine stalinienne. Le colorisme et le capisme ont par la suite progressivement représenté l'art officiel, tandis que la diversité des pratiques artistiques, si celles-ci ne portaient pas directement sur le contexte politique, a été globalement tolérée par la Pologne socialiste jusqu'à l'état de guerre décrété en décembre 1981. Cette liberté relative dont les acteurs de la scène artistique ont bénéficié leur a permis de s'intéresser, depuis la Pologne, aux productions artistiques internationales par le biais de publications et de contacts établis avec l'étranger, certains d'entre eux ayant eu l'autorisation de voyager. Cette tolérance fut cependant régulièrement ébranlée par les événements socio-politiques qui ont agité le pays : la révolte étudiante de 1968, la campagne antisémite menée par l'État à l'occasion de la répression des mouvements ouvriers en 1970 ou à l'issue du mouvement Solidarność dans les années 1980, sans compter les actions ponctuelles de la censure contre les pratiques artistiques trop ostensiblement politiques. Ainsi, à travers les études sur l'art polonais, se dessine une scène artistique polonaise originale bien plus libre et ouverte sur la scène internationale que la plupart de ses voisins socialistes durant la guerre froide et bien moins contrainte par le pouvoir en place que ne l'ont été les cercles littéraire ou philosophique polonais.

La relation du pouvoir politique aux ouvres durant la guerre froide a néanmoins suscité l'intérêt d'historiens de l'art, dont plusieurs d'ailleurs ont posé les jalons de la discipline en Pologne, et ont reconnu l'importance du sujet comme un enjeu capital notamment dans le débat des années $1970^{3}$. Voyant dans les œuvres les témoins du positionnement des artistes dans la société civile, ils se sont attachés à en révéler l'éventuel potentiel critique au regard du contexte polonais. Leurs écrits, incontournables pour comprendre les enjeux de l'histoire de l'art polonais de la guerre froide, constituent aujourd'hui une 
1. Numéro spécial " Pologne», Art press, $\mathrm{n}^{\circ} 71$, juin 1983. référence pour une génération plus jeune d'historiens de l'art. Loin d'une synthèse historiographique sur le sujet, c'est à partir de quelques textes exemplaires étudiés chronologiquement que nous montrerons comment les auteurs de ces textes ont abordé l'art polonais de la guerre froide à travers ses liens avec le pouvoir.

\section{L'art infiltré par le pouvoir}

Si les artistes polonais ont connu une relative liberté d'expression dès les premiers instants du dégel en 1954, il était néanmoins proscrit, durant le régime socialiste, d'exprimer ouvertement des positions politiques à travers une pratique artistique. En 1983, l'exposition Présences polonaises, organisée au Centre Georges Pompidou ${ }^{4}$, se gardait bien d'aborder directement les sujets politiques, contrairement à d'autres expositions d'art étranger contemporain comme Art Allemagne Aujourd'hui, présentée en 1981 au Musée d'art moderne de la Ville de Paris. Face à l'enfermement imposé alors à la Pologne par le général Jaruzelski, Ryszard Stanisławski, directeur du musée de Łódź, a occulté toute référence aux questions politiques dans le catalogue : il a préféré se concentrer sur les liens entre les pratiques contemporaines polonaises et les avantgardes polonaises d'avant-guerre, et leur place au sein des tendances artistiques internationales, soulignant par ce biais l'importance et l'indépendance de cette scène artistique. De même, dans son texte " Absences Polonaises " en réponse à Présences polonaises, Mieczesław Porębski ${ }^{5}$, alors professeur à l'université Jagellon de Cracovie, s'interrogeait sur l'éventualité qu'à la chute de "l'utopie universelle " succède " une conjoncture favorable aux particularismes des langages indirects $"{ }^{6}$, mais il ne traitait pas directement des enjeux politiques du moment. Au contraire, dans un numéro spécial d'Art press de 1983, les Polonais émigrés en France exprimaient ouvertement leur inquiétude quant aux incidences potentielles du pouvoir en place sur le choix des œuvres présentées ${ }^{7}$ (fig. 1).

Les méthodes pour appréhender les liens entre l'art et le pouvoir en Pologne ne peuvent être comprises si le lecteur n'a pas en tête qu'elles s'articulent aux importants changements qu'a connus l'écriture de l'histoire

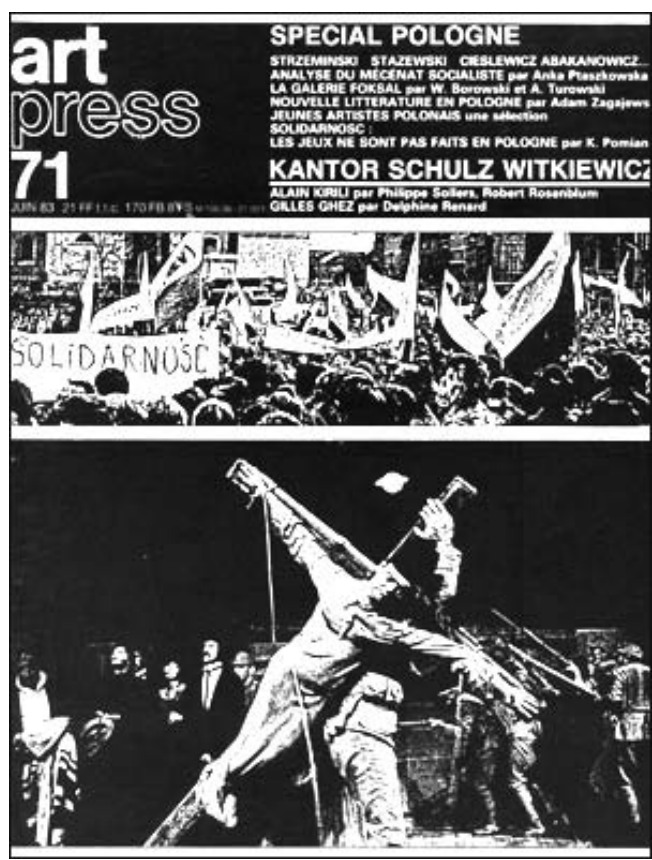

des pays socialistes depuis les années 1950 . Le totalitarisme comme modèle explicatif issu des sciences politiques a d'abord analysé les ambitions, les stratégies et les directives du pouvoir politique en place et de ses dirigeants; la société civile ne faisait pas l'objet d'études nuancées et apparaissait inféodée au pouvoir. À la fin des années 1970, la définition du post-totalitarisme issue des réflexions politiques de Václav Havel, particulièrement dans son texte Le Pouvoir des sans-pouvoir ${ }^{8}$, propose de montrer comment les individus dans les sociétés socialistes, par la perte de tout espoir de changement, se soumettent au régime et contribuent à perpétuer le système en place. Dès les années 1990, la démarche socio-historique a apporté de nouvelles nuances fondamentales grâce à des recherches micro-historiques, pour en finir avec la vision d'un bloc de l'Est uniforme et pour souligner la diversité d'appréciation, de mises en œuvre et de respect des directives du pouvoir dans les sociétés socialistes selon les pays, les époques et les milieux sociaux ${ }^{9}$. Ces différentes analyses du pouvoir et de ses incidences sur les sociétés socialistes ont eu d'importantes répercussions sur les études d'histoire de l'art.

En 1984, Andrzej Turowski a prononcé une communication sur l'art comme objet des manipulations du pouvoir devant l'association des 
historiens de l'art à Varsovie. Le texte a été publié en polonais en $1987^{10}$ et en français un an plus tard dans le premier numéro de la revue Ligeia, dirigée par Giovanni Lista ${ }^{11}$. Turowski, d'abord critique d'art a rejoint en 1970 un lieu essentiel de l'art indépendant polonais, la galerie Foksal de Varsovie, dont il est devenu l'un des principaux théoriciens. Il a par la suite été nommé professeur d'histoire de l'art à l'université de Poznań, avant de rejoindre en 1983 l'université de Clermont-Ferrand, puis celle de Dijon ${ }^{12}$. Dans le texte français qui traite la politique culturelle du pouvoir politique communiste en Pologne, Turowski ne prétend pas présenter un art aux ambitions pures qui serait victime du pouvoir ; il analyse les ressorts par lesquels le pouvoir a infiltré l'art, mais sans appuyer sa démonstration sur des exemples concrets.

Selon Turowski, rien ne peut échapper au conditionnement idéologique. Il estime que les artistes ont perdu toute autonomie et toute capacité de choix personnel, faisant ainsi lointainement écho à la façon dont Havel décrit les intrusions du pouvoir dans la vie quotidienne ${ }^{13}$. Pour décrire le climat en Pologne à cette époque, Turowski propose la notion d' "idéose ", qu'il définit " comme un espace de pensée et de systèmes, mais un espace où les choix individuels se révèlent à la lumière des stratégies politiques dominantes. Il s'agit d'un espace imprégné d'idéologie qui restreint toute manifestation de pensée souveraine. Quel que soit l'argument de cette perspective idéologique imposée et omniprésente - 'nécessité historique', 'raison d'État', 'consensus général' ou 'seul objectif juste' - il est formulé du point de vue de l'instance politique et de son primat sur les décisions individuelles ${ }^{14}$. Face à une Pologne fermée sur elle-même, Turowski veut remettre en cause l'autoritarisme idéologique qui a privé les individus de raisonnement et de sens critique, et qui a dominé sans discontinuité pendant la période de 1945 à 1980. En insistant sur cette continuité, il vise à condamner la République populaire de Pologne dans son ensemble afin de prévenir toute idéalisation et tout relativisme de la réalité politique en dépit d'une certaine ouverture du pays jusqu'au début des années 1980.
Il ressort de l'analyse de Turowski que la production artistique polonaise réalisée durant la République populaire de Pologne a été entachée de sa compromission avec l'idéologie régnante, qui l'a transformée en un support de propagande. Le pouvoir en place est ainsi davantage acteur que les artistes eux-mêmes, dont la culture, les ambitions personnelles, les ambiguïtés et les débats ne font pas l'objet d'un traitement particulier. Le lecteur en retire l'image d'une politique omnipotente face à une société uniforme, sans contours distincts, dans laquelle rien ne pourrait contrebalancer l'instrumentalisation par le pouvoir politique. Ce texte, qui est très ancré dans le contexte dans lequel il a été écrit, a marqué l'histoire de l'art polonaise.

\section{L'art comme action politique}

Après l'effondrement du bloc socialiste et la courte liesse qui s'en est suivie, les questions du traitement historique de la période de la guerre froide, de la société civile et des individus dans le système socialiste se sont rapidement posées. L'ouverture des archives des services secrets a attisé les suspicions et donné lieu à de rapides condamnations, avant que la remise en contexte et le travail interprétatif d'historiens ne permettent progressivement une meilleure compréhension de la période ${ }^{15}$. Parallèlement, le remplacement rapide d'un système politique par un autre a suscité des questionnements sur la validité des modèles importés. Piotr Piotrowski ${ }^{16}$, professeur au département d'histoire de l'art de l'université Adam Mickiewicz de Poznań, qu'il a dirigé de 1999 à 2008, spécialiste de l'art polonais de la seconde moitié du $\mathrm{XX}^{\mathrm{e}}$ siècle s'est engagé dans cette entreprise dans son ouvrage paru en 1999, Znaczenia Modernizmu ${ }^{17}$. Il a repris ensuite les principaux arguments de cette synthèse de référence consacrée à l'art polonais depuis 1945 dans son autre ouvrage intitulé Awangarda w cieniu Jałty ${ }^{18}$, qui traite de la production artistique des pays satellites de l'URSS durant la guerre froide. S'interrogeant sur la place des anciens pays de l'Est dans l'historiographie des avant-gardes contemporaines, Piotrowski cherche à définir une géographie des échanges artistiques adaptée à l'analyse de l'art de cette région. Rejetant l'idée d'une globalisation absolue, il affirme au contraire l'importance des 
2. In the Shadow of Yalta, Londres, 2009, chapitre " Myths of Geometry ", p. 118119 : Kajetan Sosnowski, Fourfol Picture, 1968, Varsovie, Muzeum Narodowe w Warszawie (gauche) ; Zbigniew Gostomski, Optical Object, XXXIV, 1965, Łodź, muzeum Sztuki (droite). frontières pour la compréhension et l'analyse des échanges artistiques, tout en refusant la définition statique d'un art déterminé par son appartenance géographique. Il s'inspire des réflexions sur la géographie de l'art de Thomas DaCosta Kaufmann menées sur la même aire géographique entre le $\mathrm{XV}^{\mathrm{e}}$ et le XVIII ${ }^{\mathrm{e}}$ siècle ; des travaux de Paul Virilio sur les vecteurs d'échanges afin d'apprécier la dimension dynamique et créative des relations artistiques; et des recherches sur la notion de frame de Norman Bryson pour souligner comment des formes semblables peuvent revêtir des significations distinctes suivant le contexte ${ }^{19}$. Piotrowski estime que les œuvres ne peuvent pas être abordées comme une forme esthétique autonome mais doivent être considérées comme participant à différentes relations de pouvoir et différents processus sociaux en prise aux tensions politiques. Il identifie des moments particuliers de tension qu'il articule, pour la Pologne, autour des dates de 1949,1956 et $1968-1970^{20}$, correspondant à la mise en place du socialisme en Pologne, à la prise de pouvoir de Gomulka et aux répressions du mouvement étudiant puis ouvrier.

Dans chacun de ses livres, Piotrowski interroge les rapports formels entre les œuvres réalisées de part et d'autre du rideau de fer, et montre combien les clés de lecture développées à l'Ouest - entendu ici moins comme l'Europe que les États-Unis - paraissent inadaptées au contexte spécifique de l'Est. Comme l'a fait remarquer Luiza Nader, l'ouvrage de Piotrowski vise en particulier les théories sur le $\mathrm{XX}^{\mathrm{e}}$ siècle publiées par les critiques de la revue américaine October $^{21}$. Selon Piotrowski, la prétendue absence d'originalité du néo-constructivisme, que lui attribue Rosalind Krauss $^{22}$ est remise en cause par l'interprétation

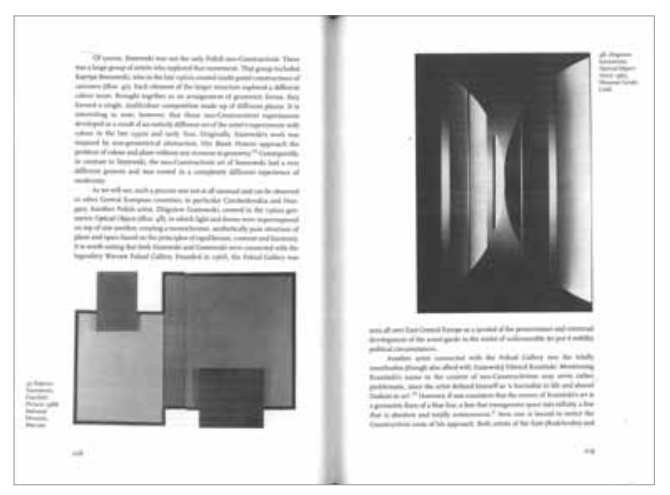

qu'en ont faite les artistes d'Europe de l'Est, et notamment les Polonais Władysław Strzemiński, Katarzyna Kobro, Henryk Stażewski, Kajetan Sosnowski et Zbigniew Gostomski, dont rien dans les œuvres n'atteste une soumission systématique à la grille de Krauss (fig. 2). De même, Piotrowski voit la réapparition de la figuration du début des années 1960, notamment par le groupe Wprost de Cracovie, non pas comme un retour à l'ordre mais comme une réaction à la réappropriation des pratiques modernistes par le pouvoir en place ${ }^{23}$. Ainsi, l'auteur évoque et contredit les conclusions de Benjamin Buchloh qui, en 1984, voyait dans la figuration le signe de l'accroissement des tendances autoritaires en politique ${ }^{24}$. La spécificité des contextes vient ainsi invalider certaines logiques interprétatives conventionnelles ${ }^{25}$. Parallèlement à la prise en considération d'espaces artistiques longtemps négligés, cette démarche, en dévoilant un autre mode de réponse artistique au pouvoir en place, incite à réinterroger plus largement ce que les œuvres révèlent de leur rapport à la politique en veillant à distinguer l'ambition des artistes d'une part et la réception de l'œuvre par la critique et par le public d'autre part ${ }^{26}$.

À cet égard, Piotrowski ne porte pas d'intérêt aux distinctions entre ces divers niveaux d'interprétations. C'est le positionnement de l'artiste face à l'appareil idéologique d'État qui retient son attention. Les artistes doivent être une force motrice et critique par rapport à la société, conscients de l'être, et capables de dénoncer. Aussi rejette-t-il les revendications d'autonomie et de liberté de l'art d'un théoricien et praticien de l'art conceptuel comme Jan Świdzinski. Piotrowski évoque le développement d'un scepticisme des artistes polonais à l'égard de l'endoctrinement et de l'engagement politique, à la suite du dégel de la fin des années 1950 qui mit en échec le système soviétique et le réalisme socialiste. Il est apparu alors un discours affirmant la liberté et l'autonomie de l'art qui s'est incarné dans des pratiques géométriques ou conceptuelles, et qui coïncidait, selon Piotrowski, avec une préférence pour le statu quo et avec une posture opportuniste des artistes. Piotrowski présente la contribution néo-constructiviste de Henrik Stażewski au symposium "Wrocław 70 " - un événement important de l'histoire de l'art 


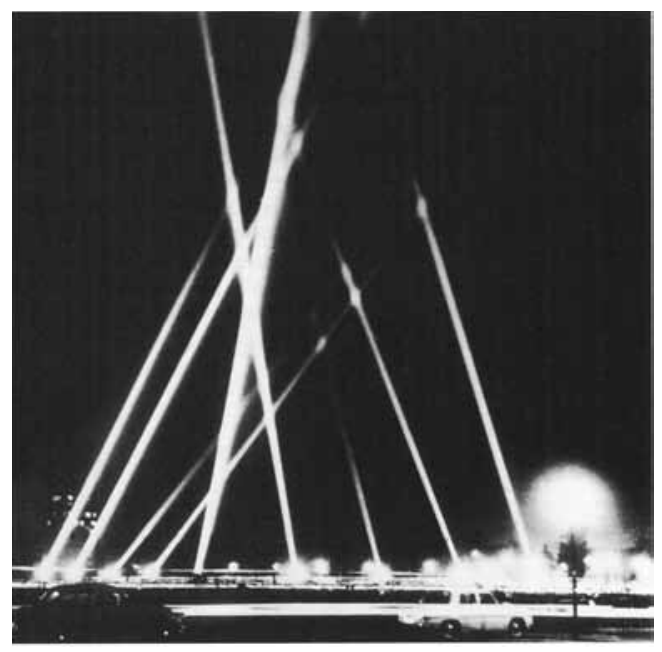

conceptuel polonais -, figure exemplaire des aspirations à un art autonome comme le résultat d'une stratégie servant les intérêts de l'artiste et les ambitions du pouvoir (fig. 3) ${ }^{27}$. Cependant le lien inextricable de l'art avec le pouvoir établi par Turowski et Piotrowski, réduit parfois l'œuvre à un objet de sciences politiques, et l'histoire de l'art à des stratégies d'artistes pour se situer, répondre et résister au système politique en place. Le livre de Piotrowski a suscité des débats houleux au cours desquels les témoins de l'époque ont contredit ses interprétations, comme l'a fait récemment Anka Ptaskowska ${ }^{28}$.

\section{Comment requalifier les liens entre art et pouvoir ?}

À la recherche de nouvelles voies interprétatives pour envisager historiquement l'art de la Pologne socialiste, la recherche en histoire de l'art contemporain polonais fait face à de nombreuses questions méthodologiques ${ }^{29}$. Aborder les liens qui unissent les pratiques artistiques au pouvoir en place et questionner leurs interprétations demeurent des enjeux centraux pour la jeune génération d'historiens de l'art qui approfondit, propose de nouveaux angles d'approche, nuance ou se distancie des questions soulevées par des auteurs comme Turowski et Piotrowski. Pour illustrer la diversification des modes d'analyse, nous nous concentrerons ici sur quelques travaux portant sur la période 1960-1970.

Le thème de l'engagement politique continue à susciter l'intérêt des chercheurs, comme Marcin
Lachowski de l'université de Lublin, qui analyse les pratiques d'avant-garde des années 1960 et 1970 dans la Pologne socialiste, en se focalisant entre autres sur les galeries Foksal, Pod Moną Lisą, Permafo, Repassage et le bureau de la poésie ${ }^{30}$. Lachowski démontre que les œuvres issues de ce mouvement, qui véhiculaient une critique des institutions, ne peuvent être interprétées comme autonomes et doivent être envisagées dans un lien inextricable avec le pouvoir. Cette lecture ne contredit cependant pas le mouvement de dépolitisation du milieu artistique qui répondait au contexte lourdement idéologique de la vie publique polonaise. À travers cette analyse, Lachowski cherche à différencier les actions artistiques qui peuvent revêtir une dimension critique, éthique, morale d'un côté et l'engagement politique partisan de l'autre. Il étudie, en outre, la réception des mouvements artistiques internationaux en Pologne dans les années 1960 et 1970 et montre comment leur interprétation a été infléchie par la spécificité du contexte polonais, revenant ainsi sur les précautions à prendre avant d'établir des mises en parallèle formelles entre des œuvres venant de part et d'autre du rideau de fer, comme l'avait fait Piotrowski.

La question du pouvoir politique est tout aussi centrale dans les travaux de Luiza Nader, historienne de l'art à l'université de Varsovie, mais son ambition tient davantage à interroger les poncifs interprétatifs de l'art conceptuel des années 1970. Dans son ouvrage Konceptualizm $w P R L^{31}$, première recherche universitaire de fond consacré à ce sujet, Nader a méticuleusement retracé le contexte historique et institutionnel de création des œuvres conceptuelles d’artistes comme Włodzimierz Borowski, Jarosław Kozłowski ou encore Krzysztof Wodiczko (fig. 4). Nader porte son attention sur la réception de ces œuvres, en reconstitue la généalogie grâce à l'analyse d'une sélection de textes fondamentaux et propose des études
3. Henryk Stażewski, Composition verticale illimitée : 9 rayons de couleur dans le ciel, Wrocław, 1970.
4. Jarosław Kozłowski, Metaphysics, cat. expo., Varsovie, galerie Foksal, 1972. 
5. Filmoteka Museum, base de films du Muzeum Sztuki : Józef Robakowski, Powietrza!, 1985 (www. artmuseum.pl/ filmoteka). de cas pour revenir sur les mythes forgés au fil du temps, à l'appui des écrits de Roland Barthes et de Giorgio Agamben, comme celui de la « mort de l'auteur ". Pour examiner le rapport des pratiques conceptuelles aux institutions et pour analyser la façon dont la critique de l'époque a associé ces œuvres à la sphère du pouvoir, Nader recourt à des méthodes issues de la psychanalyse et à la notion d'hétérotopie développée par Michel Foucault.

$C^{\prime}$ est en insistant sur le caractère visuel des œuvres que Łukasz Ronduda, historien de l'art et conservateur au centre d'art contemporain Zamek Ujazdowski à Varsovie, dans Polish Art of the $70 \mathrm{~s}^{32}$, réalisé en collaboration avec le photographe Piotr Uklański, écarte les jugements a posteriori sur les générations passées et propose de nouvelles interprétations. Grâce à une recherche pointue menée sur des artistes des années 1970 et dans les archives, présentée au fur et à mesure dans la revue Piktogram, Ronduda a fait émerger une scène mal connue. Polish Art of the 70s s'ouvre par le classement des pratiques conceptuelles entre post-essentialisme et pragmatisme, des catégories aux contours peu définis dont l'auteur use afin de caractériser l'attitude de l'artiste face à la société. L'essentiel de l'ouvrage est consacré à l'étude des démarches artistiques, comme en atteste la succession de portraits d'artistes organisée autour de l'analyse de leurs œuvres. La question du contexte spécifique de la République populaire de Pologne ne ressurgit qu'à la fin de l'ouvrage à travers les titres de deux chapitres : "Soc-Art, or an Attempt at Politicizing Aesthetics " et " NeoAvant-Garde Artists in the Secret Service Files ". Tout l'effort de Ronduda consiste à souligner la valeur des œuvres pour qu'elles rayonnent bien au-delà de leur contexte de production. Il affirme combien ces œuvres constituent un apport à l'histoire de l'art du $\mathrm{XX}^{\mathrm{e}}$ siècle et montre comment, par exemple, les films expérimentaux de Józef Robakowski ont renouvelé la compréhension que l'on a de la subversion en $\operatorname{art}^{33}$.

Ainsi, les jeunes chercheurs approfondissent la connaissance de la période de la guerre froide et diversifient les propositions interprétatives par différents biais méthodologiques, mais aussi par l'exploration de nouveaux sujets facilitée par la mise en place d'outils et la prise en compte d'objets d'études inédits. Les recherches récentes

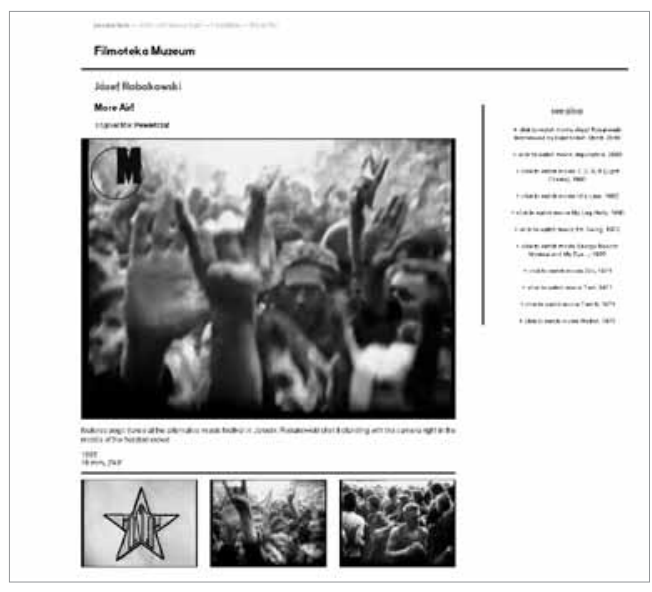

s'accompagnent d'impressionnants programmes de numérisation ${ }^{34}$, de bases de données de films (fig. 5) ${ }^{35}$, d'entretiens d'artistes ${ }^{36}$, et de textessources constituant un socle solide. À partir de ces données, les chercheurs mènent des enquêtes sur les artistes et les groupes d'artistes, et mettent progressivement à distance les enjeux politiques et mémoriels qui pèsent sur les analyses ${ }^{37}$. D'autres domaines sont toutefois encore en friche. Les chercheurs polonais ne disposent ainsi d'aucune étude fondamentale permettant de situer précisément les rapports des artistes aux manifestations du pouvoir, ni sur les institutions artistiques et leur politique après $1960^{38}$, ni sur les périodiques et sur la littérature grise ${ }^{39}$, ni sur la critique et les historiens de l'art ${ }^{40}$, ni sur l'enseignement artistique.

Une approche chronologique de l'articulation de l'art et du pouvoir dans l'histoire de l'art contemporain polonais permet d'apprécier les transformations qu'a connues cette thématique au fil des changements politiques, des années 1980 à aujourd'hui. La volonté de requalifier la nature des liens entre art et pouvoir répond à la curiosité d'interroger la place du politique dans l'art de la seconde moitié du $\mathrm{XX}^{\mathrm{e}}$ siècle. Cette démarche intéresse autant l'Est que l'Ouest et nécessite d'avancer prudemment et avec discernement pour distinguer les thèmes politiques et leur interprétation selon les pays, pour apprécier les ambitions et les intentions des artistes comme de la critique, et pour reconnaître la présence de ces aspirations dans les œuvres et leur effet aujourd'hui. Ces débats invitent à des rencontres entre chercheurs européens 
pour nouer un dialogue sur des méthodes croisées autour de questions communes, à la condition de poser clairement les traditions historiographiques vis-à-vis desquelles chacun se situe et de ne pas tomber dans des évidences interprétatives ${ }^{41}$. Ainsi peut-on croire en l'évolution de l'étude sur l'art en Europe durant la guerre froide et ne pas céder aux propos de Peter Schjeldahl, qui expliquait dans le catalogue de l'exposition Mirostav Batka en 1993 que les artistes de l'Est devaient apprendre à parler le langage de l'Ouest avant que celui-ci ne puisse en estimer la nouveautét ${ }^{42}$.

1. Europa, Europa: Das Jahrhundert der Avantgarde in Mittel- und Osteuropa, (cat. expo., Bonn, Kunst- und Ausstellungshalle der Bundesrepublik Deutschland, 1994), Ostfildern, 1994 ; Der Riss im Raum: Positionen der Kunst seit 1945 in Deutschland, Polen, der Slowakei und Tschechien, (cat. expo., Berlin, MartinGropius-Bau/Varsovie, Galeria Zaçheta, 1995), Berlin, 1994 ; Les Promesses du passé : une histoire discontinue de l'art dans l'exEurope de l'Est, (cat. expo., Paris, Musée national d'Art moderne, Centre Georges Pompidou, 2010), Paris, 2010.

2. Anda Rottenberg, "L'art en Pologne, 1945-2004", dans Le XX siècle dans l'art polonais, Paris, 2004, p. 31-83. voir du même auteur, Art from Poland 1945-1996, Varsovie, 1997 ; Sztuka w Polsce, 1945-2005, Varsovie, 2005. Voir aussi The Power of Fantasy: Modern and Contemporary Art from Poland, David Crowley éd., (cat. expo., Bruxelles, Palais des Beaux-Arts, 2011), Bruxelles/Munich, 2011.

3. Klara Kemp-Welch, "Polish Conceptualism: Expanded, Politicized, Contested (Book Review) " dans Artmargins, $1^{\mathrm{er}}$ mars 2010, publié en ligne : www.artmargins.com/ index.php/4-books/562-polish-conceptualism-expandedpoliticized-contested-book-review.

4. Présences polonaises : l'art vivant autour du musée de Łódz, Witkiewicz, constructivisme, les contemporains, (cat. expo., Paris, Musée national d'Art moderne, Centre Georges Pompidou, 1983), Paris 1983.

5. Mieczysław Porębski, "Absences polonaises ", dans Cahiers du Musée national d'art moderne, 12, 1983, p. 194-203.

6. Porębski, 1983, cité n. 5, p. 200.

7. Voir les articles de Krzystof Pomian, "Les jeux ne sont pas faits en Pologne ", et Anka Ptaszkowska "À l'ombre du bienfaiteur. Une analyse du mécénat socialiste ", dans Artpress, juin 1983, p. 4-6 et p. 22-24.

8. Václav Havel, Le Pouvoir des sans-pouvoir, Paris, 1989 [éd. orig. : Moc bezmocných, Prague, 1978]

9. Au sujet des débats historiographiques sur le modèle totalitaire et les systèmes politiques de l'ancienne Europe de l'Est, voir Enzo Traverso, Le Totalitarisme : Le XX' siècle en débat, Paris, 2001 ; Michel Christian, Emmanuel Droit, "Écrire l'histoire du communisme : l'histoire sociale de la RDA et de la Pologne communiste en Allemagne, en Pologne et en France ", dans Genèses, 61/4, 2005, p. 118-133. Au sujet de la socio-histoire, voir Sandrine Kott, « Pour une histoire sociale du pouvoir en Europe communiste : introduction thématique", dans Revue d'histoire moderne et contemporaine, 49/2, 2002, p. 5-23 ; Michel Christian, Sandrine Kott, "Introduction. Sphère publique et sphère privée dans les sociétés socialistes. La mise à l'épreuve d'une dichotomie ", dans Histoire@Politique, 1/7, 2009, publié en ligne : $w w w . h i s t o i r e-p o l i t i q u e . f r / i n d e x . p h p ?$ numero $=07 \delta$ rub=dossiersitem $=71$.

10. Andrzej Turowski, "Polska ideoza ", dans Sztuka polska po 1945 r. Materiaty sesji Stowarzyszenia Historyków Sztuki, Warszawa listopad 1984, Varsovie, 1987, p. 31-38.

11. Andrzej Turowski, "L'idéose' polonaise. La politique culturelle du pouvoir communiste en Pologne 1945 1981 ", dans Ligeia, 1, 1988, p. 30-35.

12. S'ils sont écrits le plus souvent en polonais, plusieurs des textes de Turowski ont été traduits et comptent parmi la trop rare littérature en langue française concernant l'art polonais et la région d'Europe centrale plus largement. Voir notamment Andrzej Turowski, « ... L'art organisant la vie et ses fonctions... ", dans Présences polonaises..., 1983, cité n. 4, p. 140145 ; Existe-t-il un art de l'Europe de l'Est?, Saint-Étienne, 1986 ; « De la forme pure à la figure de la mort. Concepts théoriques de l'avant-garde polonaise au XX $\mathrm{X}^{\mathrm{e}}$ siècle ", dans Le XXe siècle dans l'art polonais, Paris, 2004, p. 15-30.

13. Havel, 1989, cité n. 8 .

14. Turowski, 1988, cité n. 11.

15. Voir par exemple Andrzej Friszke, Anatomia Buntu: Kuroń, Modzelewski i komandosi, Cracovie, 2010.

16. Piotr Piotrowski a également été conservateur d'art contemporain au Muzeum Narodowe à Poznań, et a dirigé le Muzeum Narodowe à Varsovie. Éditeur du journal Artium Quaestiones, il est l'auteur de nombreux ouvrages qui examinent de la scène artistique de la seconde moitié du $\mathrm{XX}^{\mathrm{e}}$ siècle et du début du XXI siècle, plusieurs de ses textes étant accessible en anglais.

17. Piotr Piotrowski, Znaczenia Modernizmu, Poznań, 1999.

18. Piotr Piotrowski, In the Shadow of Yalta: Art and the Avant-Garde in Eastern Europe, 1945-1989, Londres, 2009 [éd. orig. : Awangarda w cieniu Jatty, Poznań, 2004].

19. Thomas DaCosta Kaufmann, Court, Cloister and City: The Art and Culture of Central Europe, 1450-1800, Londres, 1995 ; Paul Virilio, Open Sky, Londres, 1997 ; Norman Bryson, "Art in Context ", dans Studies in Historical Change, Ralph Cohen éd., Charlottesville, 1992.

20. Piotrowski, 1999, cité n. 17.

21. Luiza Nader et al., "'Jałta' i cień awangardy. Dyskusja redakcyjna wokół książki Piotra Piotrowskiego 'Awangarda w cieniu Jałty' ", dans Obieg, 25 août 2006, publié en ligne : www.obieg.pl/rozmowy/5705.

22. Voir dans Piotrowski, 2009, cité n. 18, p. 139-141. Piotrowski fait référence à Rosalind Krauss, The Originality of the Avant-Garde and Other Modernist Myths, Cambridge, 1985, p. 160 et p. 9-22.

23. Piotrowski, 1999, cité n. 17, p. 92-116. 
24. Voir dans Piotrowski, 2009, cité n. 18, p. 172-173. Piotrowski fait référence à Benjamin Buchloh dans " Figures of Authority, Ciphers of Regression: Notes on the Return of Representation in European Painting ", dans Marcia Tucke, Brian Wallis, Art after Modernism: Rethinking Representation, New York/Boston, 1984, p. 107-135.

25. Piotrowski, 2009, cité n. 18, p. 25-26.

26. Certains travaux scientifiques précisent les biais par lesquelles les artistes eurent des ambitions sociales et politiques, en s'attachant à dissocier les interprétations de la critique, les aspirations des artistes ou ce que le recul donne à voir des œuvres. Les articulations entre la production artistique, sa diffusion par la critique et son appropriation par le politique ont été examinées plus précisément, voir par exemple Jill Carrick, "Le Nouveau Réalisme : un détournement de la profusion des choses ", dans Le Nouveau Réalisme, (cat. expo., Paris, Grand Palais, 2007), Paris, 2007, p. 176-181 ; Steffen Dengler, Die Kunst der Freiheit? Die westdeutsche Malerei im Kalten Krieg und im wiedervereinigten Deutschland, Munich, 2010. L'étude du traitement de l'art par les critiques, les expositions et les politiques culturelles souligne l'écart ou l'adéquation entre les discours de certains artistes et leurs ambitions, voir notamment en France Maïté Vissault, Der Beuys Komplex : l'identité allemande à travers la réception de l'auvre de Joseph Beuys (1945-1986), Dijon, 2010 ; Richard Leeman, Le Critique, l'art et l'histoire : de Michel Ragon à Jean Clair, 1959-1972, Rennes, 2010 ; Antje Kramer, L'Aventure allemande du Nouveau Réalisme : réalités et fantasmes d'une néoavant-garde européenne (1957-1963), Dijon, à paraître.

27. Voir à ce sujet l'analyse de la participation de Stażewski au symposium Wrocław 70, dans Piotrowski, 2009, cité n. 18.

28. Anka Ptaszkowska, "'Wóz albo przewóz' - to nie jest pytanie ", dans Obieg, 13 février 2011, publié en ligne : www.obieg.pl/felieton/20279.

29. Andrzej Szczerski, Tomasz Gryglewicz éd., Sztuka w okresie PRL-u. Metody i przedmiot badań, Cracovie, 1999. Cet ouvrage réunit des articles questionnant les enjeux méthodologiques qui se posent à l'écriture de l'histoire de l'art de la Pologne socialiste.

30. Marcin Lachowski, Awangarda wobec instytucji osposobach prezentacji sztuki w PRL-u, Lublin, 2006.

31. Luiza Nader, Konceptualizm w PRL, Varsovie, 2009.

32. Łukasz Ronduda, Polish Art of the 70s, Jelenia Góra éd. Varsovie, 2009.

33. Eukasz Ronduda, "Subversive Strategies in the Media Arts. Józef Robakowski's Found Footage and Video Scratch ", publié sur le site de la galerie Wymiany : www. exchange-gallery.pl/english/subversive.html.

34. Voir le programme du Muzeum Sztuki Współczesnej à Varsovie dans la rubrique consacrée aux archives numérisées sur le site du musée : www.artmuseum.pl/archiwa.php?l=0.

35. Voir la filmothèque du Muzeum Sztuki Współczesnej à Varsovie, www.artmuseum.pl/filmoteka ; voir aussi Film Form Archive du centre d'art contemporain du château Ujazdowski à Varsovie : http://csw.art.pl/index. php?action=archfilmslang=eng.

36. Voir notamment les entretiens parus dans Obieg, www.obieg.pl.
37. On peut rappeler ici l'importance des travaux menés par les institutions muséales, comme le musée d'art contemporain et le Centrum Sztuki Współczesnej na Zamku Ujazdowskim à Varsovie, le Muzeum Sztukide à Łódź, ou encore le Muzeum Sztuki Współczesnej (Mocak) à Cracovie. Pour les ouvrages, on peut citer à titre d'exemples, Oskar Hansen: Towards Open Form, ku formie otwartej, Jola Gola éd., (cat. expo., Varsovie, Galerie Foksal, 2005), Varsovie, 2005 ; Henryk Stażewski: ekonomia myślenia i postrzegania, Małgorzata Jurkiewicz et al., (cat. expo., Varsovie, Galerie Foksal, 2006), Varsovie, 2006 ; Edward Krasiński : les mises en scène, Sabine Breitwieser éd., (cat. expo., Vienne, Generali Foundation, 2006), Vienne/ Cologne, 2006 ; Awangarda w Bloku: avant-garde in the Bloc, Gabriela Świtek éd., Varsovie/Zurich/New York, 2009 ; Claire Bishop, Marta Dziewańska éd., Political Upheaval and Artistic Change 1968-1989, (colloque, Varsovie, 2008), Varsovie, 2009 ; Quotation Marks: Jarostaw Kozłowski, Božena Czubak éd., Varsovie, 2010 ; Małgorzata Borkowska, Akademia Ruchu: miasto - pole akcji = City - the field of action, Varsovie, 2006.

38. Pour la période antérieure à 1960, il faut mentionner l'ouvrage dirigé par Aleksander Wojciechowski, Polskie życie artystyczne $w$ latach 1945-1960: praca zbiorowa (Wrocław, 1992), particulièrement l'article d'Anda Rottenberg, "Ministerwo Kultury i Sztuki », p. 335-346.

39. Sur la critique d'art au temps du dégel voir Piotr Juszkiewicz, Od rozkoszy historiozofii do gry w nic: polska krytyka artystyczna czasu odwilży, Poznań, 2005.

40. Pour avoir un aperçu de l'écriture de l'histoire de l'art en Pologne de 1945 à 1989, voir Joanna Sosnowska, "Polska historia sztuki 1945-1989. Kwestie metodologii ", dans Urszula Jakubowska, Jerzy Myśliński éd., Humanistyka polska w latach 1945-1990, Varsovie, 2006, p. 207-229

41. Il faut saluer le travail mené dans le cadre du centre Marc Bloch pour encourager les rencontres autour de thématiques scientifiques communes grâce à l'établissement d'un atlas des échanges artistiques entre l'Est et l'Ouest durant la guerre froide, consultable sur http://art-communism.eu. Voir également le projet ERC-Starting Grant "À chacun son réel " mené au Centre allemand d'histoire de l'art. L'ambition de ce projet, à partir de la notion de "réel " qui a été convoquée par de nombreux courants tels que le nouveau réalisme, le réalisme capitaliste et le réalisme socialiste, est d'approfondir les points de rencontre entre les pratiques artistiques venues de France, des ex-Allemagne de l'Est et de l'Ouest, et de la Pologne entre 1960 et 1989 (voir www.dtforum.org/ownreality).

42. Voir Peter Schjeldahl, "Polskie haiku " dans Mirostaw Batka, (cat. expo., Varsovie, Galeria Foksal, 1993), Varsovie, 1993, p. 17.

Mathilde Arnoux, Centre allemand d'histoire de l'art marnoux@dt-forum.org

Mots-clés
art conceptuel, engagement, guerre froide,
politique artistique, Pologne

